\title{
Overcoming inefficient cellobiose fermentation by cellobiose phosphorylase in the presence of xylose
}

\author{
Kulika Chomvong ${ }^{1}$, Vesna Kordić ${ }^{2}$, Xin Li ${ }^{3}$, Stefan Bauer ${ }^{4}$, Abigail E Gillespie ${ }^{3}$, Suk-Jin Ha, ${ }^{5,6,7}$, Eun Joong Oh, \\ Jonathan M Galazka ${ }^{3}$, Yong-Su Jin ${ }^{5,6}$ and Jamie H D Cate $2,3,8^{*}$
}

\begin{abstract}
Background: Cellobiose and xylose co-fermentation holds promise for efficiently producing biofuels from plant biomass. Cellobiose phosphorylase (CBP), an intracellular enzyme generally found in anaerobic bacteria, cleaves cellobiose to glucose and glucose-1-phosphate, providing energetic advantages under the anaerobic conditions required for large-scale biofuel production. However, the efficiency of CBP to cleave cellobiose in the presence of xylose is unknown. This study investigated the effect of xylose on anaerobic CBP-mediated cellobiose fermentation by Saccharomyces cerevisiae.
\end{abstract}

Results: Yeast capable of fermenting cellobiose by the CBP pathway consumed cellobiose and produced ethanol at rates $61 \%$ and $42 \%$ slower, respectively, in the presence of xylose than in its absence. The system generated significant amounts of the byproduct 4-O- $\beta$-D-glucopyranosyl-D-xylose (GX), produced by CBP from glucose-1-phosphate and xylose. In vitro competition assays identified xylose as a mixed-inhibitor for cellobiose phosphorylase activity. The negative effects of xylose were effectively relieved by efficient cellobiose and xylose co-utilization. GX was also shown to be a substrate for cleavage by an intracellular $\beta$-glucosidase.

Conclusions: Xylose exerted negative impacts on CBP-mediated cellobiose fermentation by acting as a substrate for GX byproduct formation and a mixed-inhibitor for cellobiose phosphorylase activity. Future efforts will require efficient xylose utilization, GX cleavage by a $\beta$-glucosidase, and/or a CBP with improved substrate specificity to overcome the negative impacts of xylose on CBP in cellobiose and xylose co-fermentation.

Keywords: Cellobiose, Cellobiose phosphorylase, Glucopyranosyl-xylose, Inhibition, Xylose

\section{Background}

Cellulosic biofuels could make significant contributions to meet the ever-rising demand for energy. To economically produce fuels from cellulosic biomass, sugars derived from cellulose as well as hemicellulose must be utilized completely [1-3]. The co-fermentation of cellobiose derived from cellulose and xylose derived from hemicellulose allows these sugars to be consumed simultaneously [4], and may enable continuous biofuel production [5]. In this system, cellobiose and xylose are transported into engineered S. cerevisiae using a cellodextrin transporter (that is, CDT1 from Neurospora crassa) and endogenous hexose transporters, respectively $[4,6]$. Intracellular cellobiose is then

\footnotetext{
* Correspondence: jcate@lbl.gov

${ }^{2}$ Department of Chemistry, University of California, Berkeley, CA, USA

${ }^{3}$ Department of Molecular and Cell Biology, University of California, Berkeley, CA, USA

Full list of author information is available at the end of the article
}

hydrolyzed into two molecules of glucose by an intracellular $\beta$-glucosidase (NCU00130; GH1-1) [6]. At the same time, xylose is consumed by an oxidoreductive pathway, comprising xylose reductase and xylitol dehydrogenase, that converts xylose to xylulose $[7,8]$. Alternatively, xylose can be utilized by xylose isomerase, which converts xylose directly to xylulose [9]. Glucose and xylulose are then metabolized using glycolysis and the pentose phosphate pathway, respectively, resulting in ethanol production [4].

A pathway potentially better suited to the anaerobic environment of large-scale biofuels production substitutes cellobiose phosphorolysis for the hydrolytic reaction of $\beta$ glucosidase $[10,11]$. This pathway comprises cellobiose phosphorylase (CBP), which cleaves intracellular cellobiose into glucose and glucose-1-phosphate (G1P) [12]. The

Ciomed Central

(n)

(c) 2014 Chomvong et al.; licensee BioMed Central Ltd. This is an Open Access article distributed under the terms of the Creative Commons Attribution License (http://creativecommons.org/licenses/by/4.0), which permits unrestricted use, distribution, and reproduction in any medium, provided the original work is properly credited. The Creative Commons Public Domain Dedication waiver (http://creativecommons.org/publicdomain/zero/1.0/) applies to the data made available in this article, unless otherwise stated. 
phosphorolytic pathway requires one less ATP for each molecule of cellobiose to be metabolized by glycolysis. This is because glucose generated by hydrolysis of cellobiose requires two ATP molecules for hexokinase generation of glucose-6-phosphate (G6P) [13], whereas CBP uses inorganic phosphate in place of one of the ATP molecules to produce G1P. G1P can then be converted to G6P without the need for ATP by the enzyme phosphoglucomutase [14]. Under anaerobic conditions, in which glycolysis generates only two ATP molecules per glucose, increased ATP can result in increased biomass at the expense of ethanol product yield [15]. However, the phosphorolytic pathway can be engineered to perform better than the hydrolytic pathway in terms of product yield in stressful conditions like those expected in lignocellulosic hydrolysates [10].

Although the cellobiose phosphorolytic pathway has potential advantages, its efficiency, hereafter defined as incomplete or low rate of consumption, in the context of cellobiose and xylose co-fermentation is not known. Previously, cellobiose phosphorolytic pathways were combined with xylose isomerase pathways to construct anaerobic cellobiose and xylose co-fermentation systems in Saccharomyces cerevisiae and in Escherichia coli $[16,17]$. However, they are inefficient in terms of sugar consumption and ethanol production rates [16], or the systems remain to be fully optimized [17]. CBP from Ruminococcus albus NE1 (RaCBP) uses xylose as a substrate for the reverse of the phosphorolytic reaction [18]. We therefore hypothesized that the inefficiency in cellobiose and xylose co-fermentation previously observed was due to xylose interference with cellobiose consumption via CBP. The presence of xylose is unavoidable because it is a major component of hemicellulose, which has to be utilized for economical biofuel production [1-3]. We therefore tested the effect of xylose on CBP cellobiose fermentation, as well as two potential approaches to alleviate inefficient CBP-mediated cellobiose fermentation in the presence of xylose.

\section{Results}

\section{Inefficient cellobiose fermentation in the presence of xylose}

A codon-optimized CBP gene from Saccharophagus degradans (SdCBP) [10] and a mutant cellodextrin transporter encoding $N$. crassa CDT-1 (F213L) [10] were cloned into the $2 \mu$ plasmid pRS426 under the control of constitutive $\mathrm{P}_{P G K 1}$ promoters (hereafter called pCS plasmid). S. cerevisiae strain D452-2 transformed with this plasmid was used for anaerobic fermentations (Table 1). The fermentations were carried out with $80 \mathrm{~g} / \mathrm{L}$ of cellobiose as a carbon source, either with or without $40 \mathrm{~g} / \mathrm{L}$ of xylose present. The engineered strain was capable of fermenting cellobiose to ethanol in both conditions
Table 1 Strains and plasmids used in this study

\begin{tabular}{|c|c|c|}
\hline $\begin{array}{l}\text { Strains/ } \\
\text { plasmids }\end{array}$ & Characteristics & Reference \\
\hline D452-2 & MATalpha, leu2, his3, ura3, and can 1 & {$[19]$} \\
\hline SR8 & 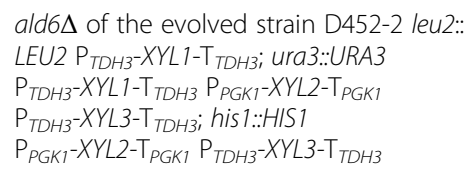 & {$[20]$} \\
\hline SR8-a & SR8 ura3 & $\begin{array}{l}\text { Courtesy of } \\
\text { Dr. Soo Rin Kim }\end{array}$ \\
\hline pCT & pRS426- $P_{P_{G K 1}-c d t-1(F 213 L)-e G F P-t_{C Y C 1}}$ & [10] \\
\hline pSd & 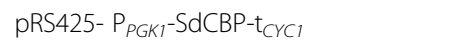 & [10] \\
\hline pCS & $\begin{array}{l}\text { pRS426-P } P_{P G K 1}-c d t-1 \text { (F213L)-eGFP-t }{ }_{C Y C I^{-}} \\
P_{P G K 1}-S d C B P-t_{C Y C 1}\end{array}$ & This study \\
\hline pET-Sd & pET302-NT/6his-SdCBP & This study \\
\hline
\end{tabular}

(Figure 1A,B). However, in the presence of xylose, the rates of cellobiose consumption and ethanol production decreased by $61 \%$ and $42 \%$, respectively (Figure $1 \mathrm{~A}, \mathrm{~B}$, Table 2). As a result, approximately $20 \mathrm{~g} / \mathrm{L}$ of cellobiose remained in the fermentation broth after 72 hours in the presence of xylose (Figure 1A), whereas all of the cellobiose was consumed within 36 hours in the absence of xylose (Figure 1A). These results indicated that the presence of xylose had a severely negative impact on cellobiose fermentation mediated by CBP.

Interestingly, in the fermentation supplied with $40 \mathrm{~g} / \mathrm{L}$ of xylose, the xylose concentration showed an initial decrease followed by a slight recovery after 36 hours (Figure 1C). Xylitol was also produced with a titer of approximately $9 \mathrm{~g} / \mathrm{L}$ at 72 hours (Figure 1D). These results suggest that approximately half of the xylose transported into the cell was reduced to xylitol but the rest remained unaccounted for.

\section{In vitro and in vivo production of glucopyranosyl-xylose}

To determine the fate of xylose that was unaccounted for by xylitol production, xylose and G1P were used as substrates for the reverse reaction catalyzed by purified SdCBP. Chromatograms of the reaction analyzed by ion chromatography showed a decrease in xylose and G1P concentration along with the appearance of a new peak (Figure 2A). Analysis of the reaction by mass spectrometry (MS) identified the molecular mass of the product to be $312 \mathrm{~g} / \mathrm{mol}$, consistent with that of 4-O- $\beta$-D-glucopyranosyl-D-xylose (GX) (Figure 2B, Additional file 1: Figure S1A). Tandem mass spectrometry (MS-MS) further indicated that the product comprised one hexose unit and one pentose unit (Additional file 1: Figure S1B). These results suggested that the in vitro reverse reaction of SdCBP produced a GX dimer when xylose and G1P were provided as substrates. 

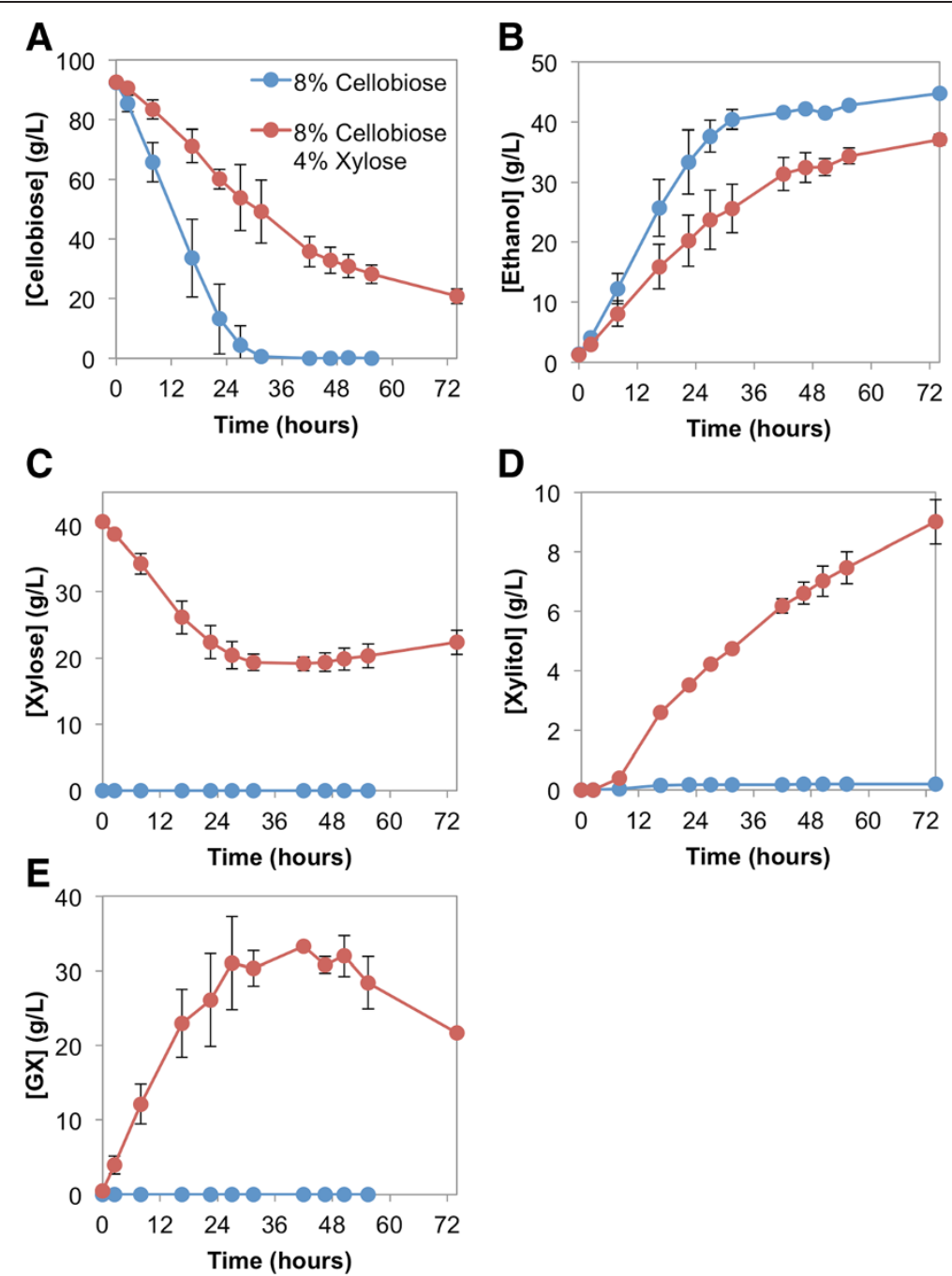

Figure 1 Fermentation profile of engineered strain D452-2 in the presence and absence of xylose. S. cerevisiae strain D452-2 was transformed with the pCS plasmid, encoding cellodextrin transporter cdt1-F213L and SdCBP. Anaerobic fermentations were supplied with $80 \mathrm{~g} / \mathrm{L}$ cellobiose in the presence (red dots) and absence (blue dots) of $40 \mathrm{~g} / \mathrm{L}$ xylose. Extracellular concentrations of (A) cellobiose, (B) ethanol, (C) xylose, (D) xylitol and (E) glucopyranosyl-xylose are shown. Values and error bars represent the means and standard deviations of two independent biological replicates.

Using GX synthesized in vitro as a standard, GX was detected in the fermentation broth when cellobiose and xylose were supplied to yeast engineered with the CBP cellobiose consumption pathway (Figure 1E). Interestingly, the concentration of GX initially increased but started to decrease after 36 hours, with its highest concentration reaching approximately
$30 \mathrm{~g} / \mathrm{L}$ (Figure 1E). Extracellular concentrations of xylitol and GX combined accounted for $88 \%$ to $100 \%$ of the imported xylose (Additional file 1: Figure S2). Thus, yeast utilizing cellobiose by the CBP consumption pathway formed GX from intracellular xylose and G1P, in addition to converting some of the imported xylose to xylitol.

Table 2 Fermentation parameters

\begin{tabular}{|c|c|c|c|}
\hline Strain & Media & Cellobiose consumption rate $(\mathrm{g} / \mathrm{L} \cdot \mathrm{h})$ & Ethanol production rate $(g / L \cdot h)$ \\
\hline D452-2 & Cellobiose & $3.6 \pm 0.05$ & $1.5 \pm 0.03$ \\
\hline D452-2 & Cellobiose + xylose & $1.4 \pm 0.04$ & $0.85 \pm 0.02$ \\
\hline SR8-a & Cellobiose & $3.7 \pm 0.09$ & $1.5 \pm 0.04$ \\
\hline SR8-a & Cellobiose $+x y l o s e$ & $2.9 \pm 0.08$ & $1.6 \pm 0.04$ \\
\hline
\end{tabular}




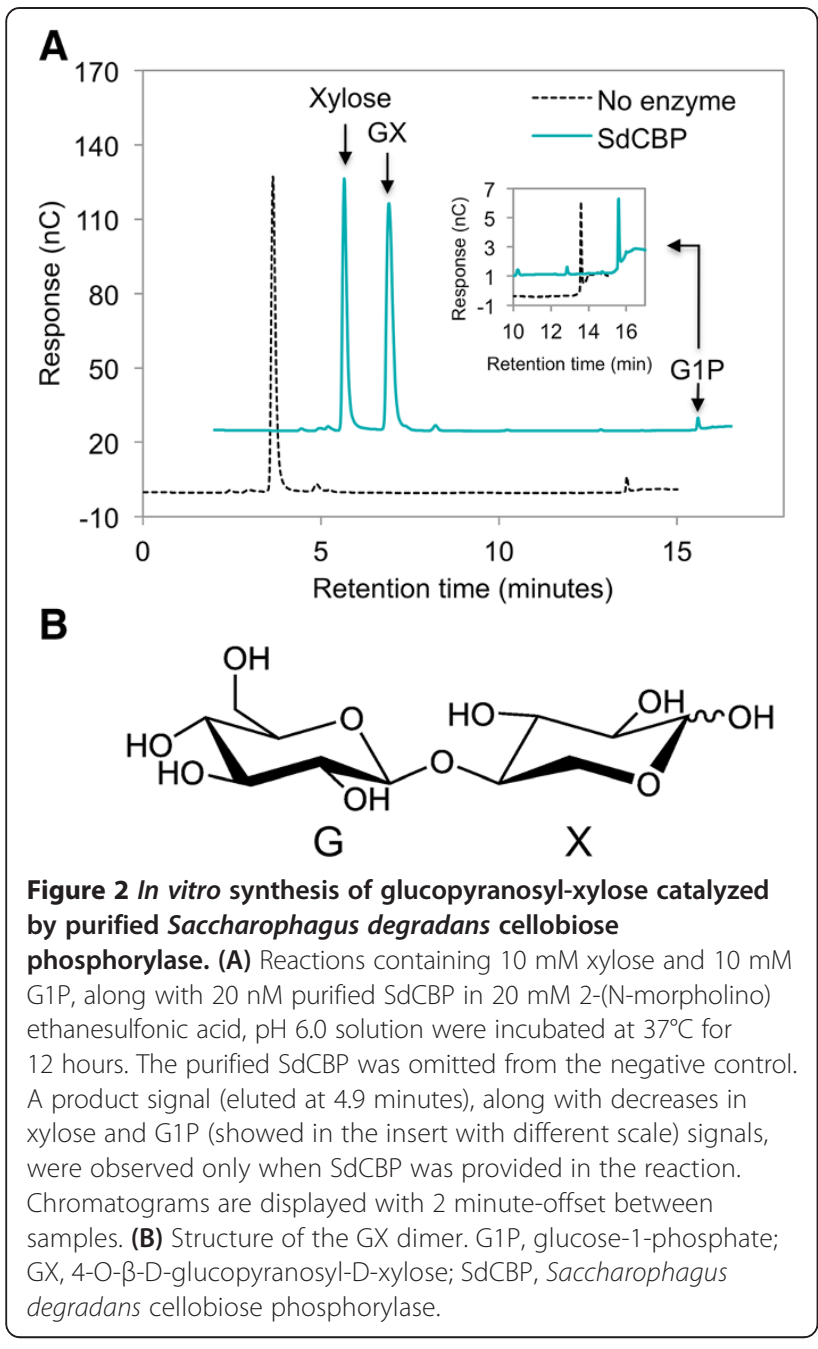

Competition assays identified xylose as a mixed-inhibitor To investigate the inhibitory effect of xylose on SdCBP activity, the catalytic properties of SdCBP were determined in the presence of varying xylose concentrations (Figure 3), at the time points preceding the production of $\mathrm{GX}$ (Additional file 1: Figure S3). Initial rates of cellobiose phosphorolysis were calculated from the amount of G1P produced at different cellobiose concentrations (Figure 3A). As the concentration of xylose increased, the apparent maximal rate $\left(\mathrm{V}_{\text {max,app }}\right)$ linearly decreased while the apparent substrate concentration at which the reaction rate is half of $V_{\text {max,app }}\left(K_{M, a p p}\right)$ linearly increased (Figure $\left.3 A, B\right)$. This result indicated that the apparent affinity of CBP for cellobiose and its maximal phosphorolytic rate for cellobiose were inversely proportional to the xylose concentration, identifying xylose as a mixed-inhibitor for the cellobiose phosphorolysis reaction (Figure 3B). The negative impact of xylose on SdCBP phosphorolytic activity may therefore contribute to the decrease in cellobiose consumption rate observed phenotypically in the above fermentations (Figure 1A), in addition to the production of GX.

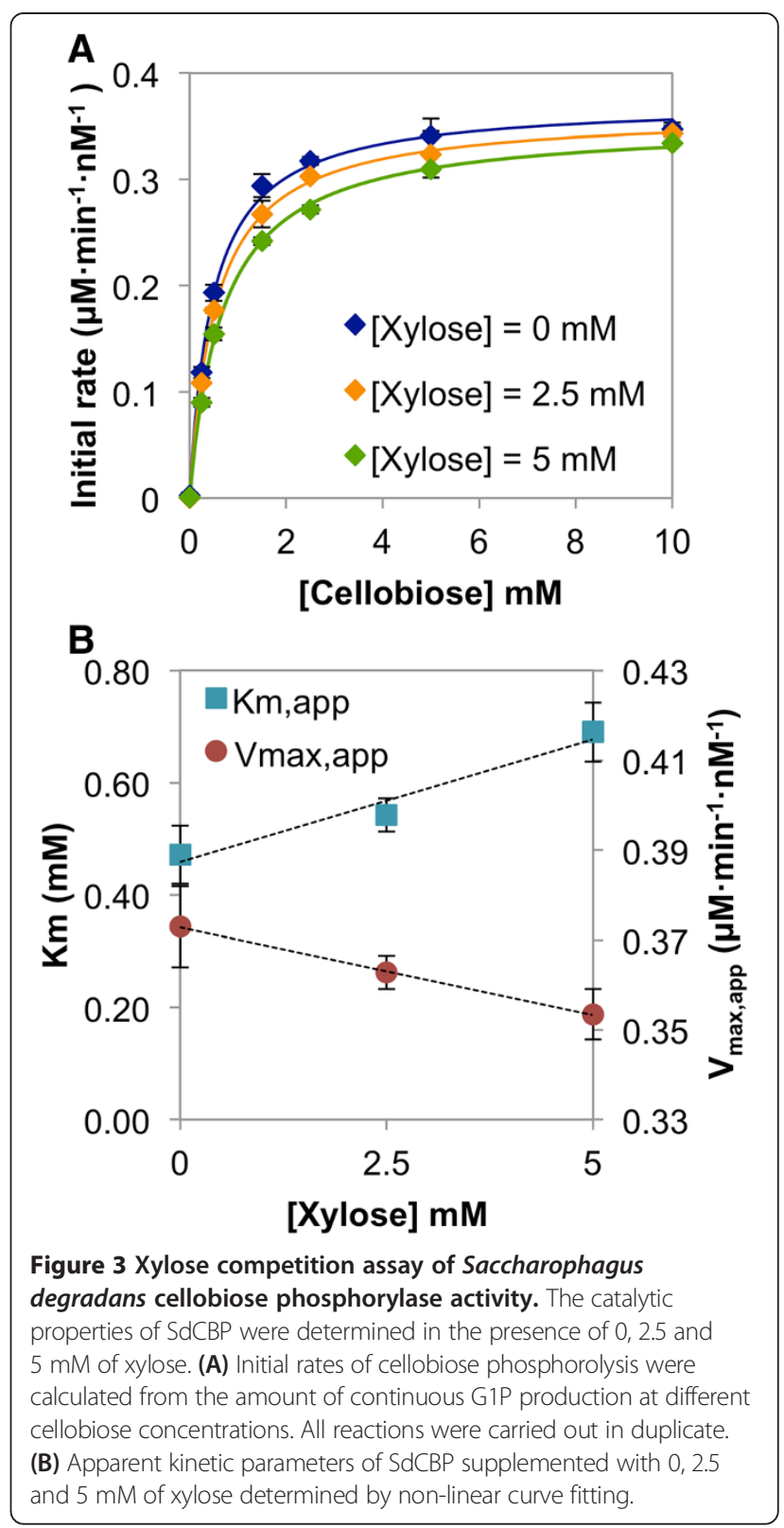

Reduced glucopyranosyl-xylose formation in an efficient xylose utilizing strain, SR8-a

Strain SR8-a is an engineered S. cerevisiae strain capable of rapid xylose fermentation [20]. We wondered whether rapid xylose utilization, which would maintain a lower intracellular concentration of xylose, could mitigate formation of GX by CBP. Strain SR8-a (Table 1) and strain D452-2 used above, which lacks a xylose-utilization pathway, were transformed with the pCS plasmid and fermentations were carried out with $80 \mathrm{~g} / \mathrm{L}$ of cellobiose as a carbon source, with or without $40 \mathrm{~g} / \mathrm{L}$ of xylose present. In the absence of xylose, the cellobiose consumption and ethanol production profiles of the engineered D452-2 and SR8-a strains were equivalent (Additional file 1: 
Figure S4). Notably, in the presence of xylose, the cellobiose consumption rate of the SR8-a strain was two-fold higher than that of the D452-2 strain (Figure 4A, Table 2). The SR8-a strain completely consumed cellobiose in 40 hours and xylose in 24 hours (Figure 4A,C). In the SR8-a background, GX was produced at a lower concentration, at a slower rate, and started to decrease after 16 hours, in comparison to 48 hours in the D452-2 strain
(Figure 4E). In addition, the engineered SR8-a strain produced less xylitol and more ethanol than the D452-2 strain (Figure 4B,D). This result suggested that efficient xylose utilization reduced the formation of GX and increased the CBP-mediated cellobiose consumption rate in the presence of xylose.

The cellobiose consumption rate of the SR8-a strain supplemented with xylose was not as rapid as that of the
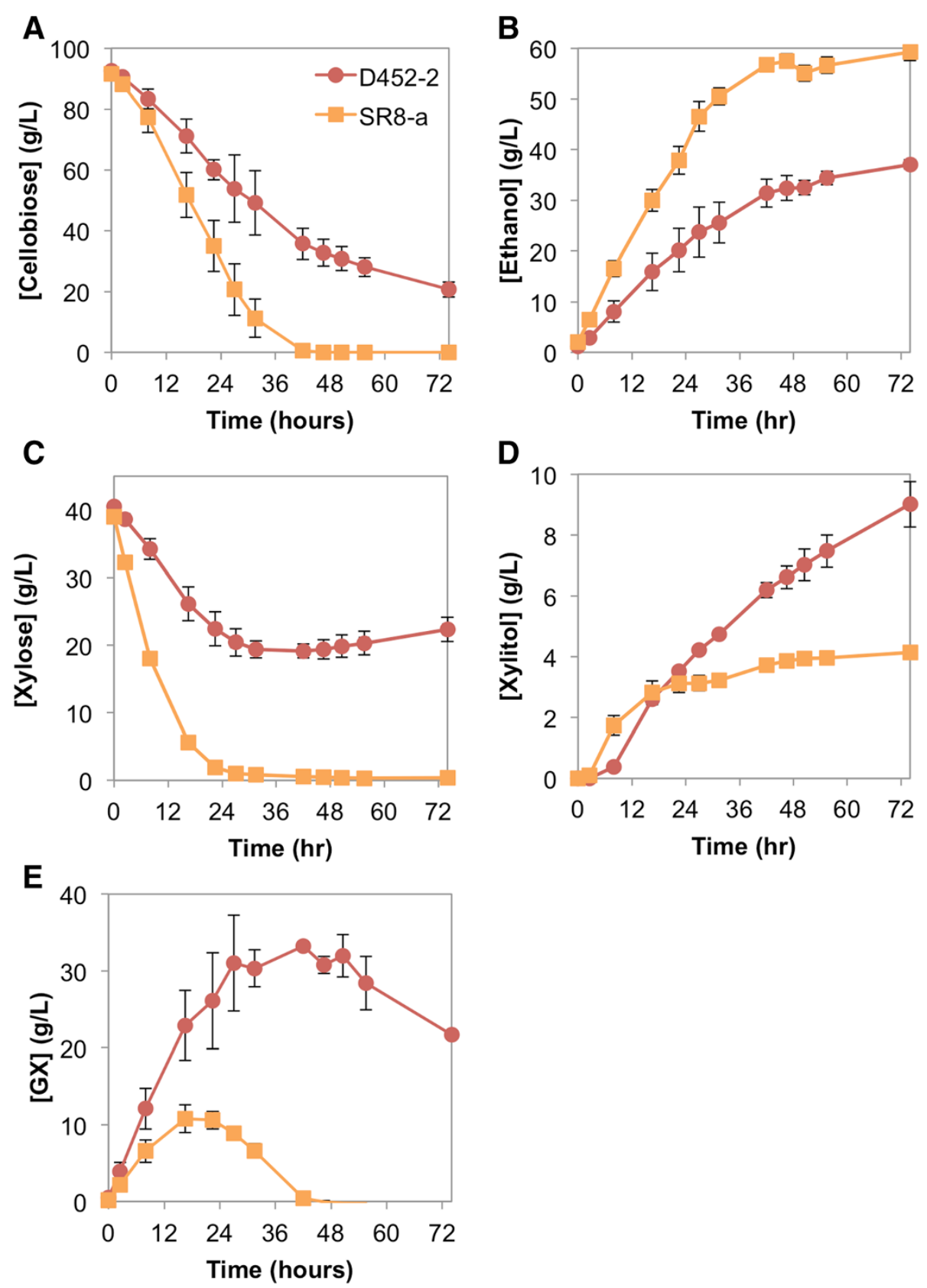

Figure 4 Fermentation profile of engineered D452-2 and SR8-a strains supplemented with cellobiose and xylose. S. cerevisiae D452-2 (red circle) and SR8-a (orange square) strains were transformed with the pCS plasmid. Anaerobic fermentations were supplied with $80 \mathrm{~g} / \mathrm{L}$ cellobiose and $40 \mathrm{~g} / \mathrm{L}$ xylose. Extracellular concentrations of (A) cellobiose, (B) ethanol, (C) xylose, (D) xylitol and (E) glucopyranosyl-xylose are shown. Values and error bars represent the means and standard deviations of two independent biological replicates. 
D452-2 strain with no xylose present, showing a $22 \%$ decrease (Figure 1A, Figure 4A, Table 2). However, the negative effect of xylose on cellobiose consumption was alleviated in comparison to the $61 \%$ decrease in cellobiose consumption rate with the D452-2 strain in the presence of xylose (Figure 1A).

\section{Cleavage of glucopyranosyl-xylose by intracellular $\beta$-glucosidase and $\beta$-xylosidase}

Given that the GX formed by CBP in the reverse phosphorolytic reaction should have a $\beta-1,4$ linkage, we wondered whether GX might serve as a substrate for either an intracellular $\beta$-xylosidase (NCU01900) or the intracellular $\beta$-glucosidase (NCU00130; GH1-1) from $N$. crassa. Although the amount of GX did not change significantly when the $\beta$-xylosidase or no enzyme was used (Figure 5 ), the $\beta$-glucosidase GH1-1 completely hydrolyzed GX, as indicated by the disappearance of the GX peak (Figure 5). Signals of the hydrolysis products, namely glucose and xylose, overlapped but the increase was detected (Figure 5). Further enzymatic analysis of $\beta$ glucosidase GH1-1 activity revealed that its maximal rate for GX cleavage was three times lower and its $K_{M}$ for $G X$ was seven-fold higher than that for cellobiose (Table 3, Additional file 1: Figure S5). These results showed that the $\beta$-glucosidase GH1-1, but not the $\beta$-xylosidase NCU01900, was capable of cleaving GX to glucose and xylose. However, cellobiose may compete with GX hydrolysis in the context of in vivo cellobiose consumption.

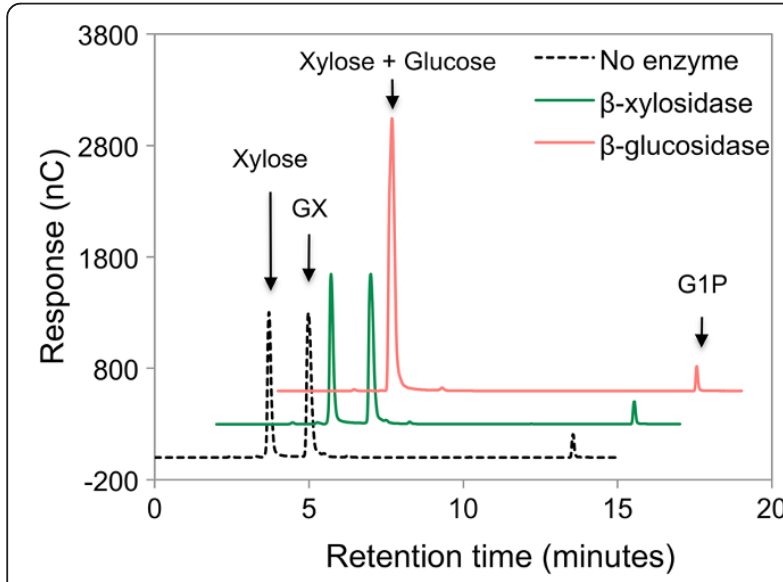

Figure 5 -xylosidase (NCU01900) and $\beta$-glucosidase (NCU00130) activities on glucopyranosyl-xylose. GX synthesized in vitro used as a substrate contained some traces of xylose and G1P. Purified $\beta$-xylosidase and $\beta$-glucosidase were incubated with GX substrate mixture in $1 \times$ PBS, pH 7.4 buffer. The purified proteins were omitted in the control reaction. Chromatograms are displayed with 2 minute-offsets between samples. GX, glucopyranosyl-xylose; G1P, glucose-1-phosphate.
Table 3 Kinetic parameters of $\beta$-glucosidase GH1-1 for glucopyranosyl-xylose and cellobiose

\begin{tabular}{lcc}
\hline Kinetic parameters & Glucopyranosyl-xylose & Cellobiose \\
\hline $\mathrm{K}_{\mathrm{M}}(\mathrm{mM})$ & $3.5 \pm 1.4$ & $0.49 \pm 0.05$ \\
$\mathrm{~V}_{\max }\left(\mu \mathrm{M} \cdot \mathrm{min}^{-1} \cdot \mathrm{nM}^{-1}\right)$ & $0.48 \pm 0.10$ & $1.3 \pm 0.04$ \\
\hline
\end{tabular}

\section{Discussion}

In this study, we identified GX production as a competing off-pathway product of cellobiose and xylose co-fermentation when cellobiose is consumed using a CBP-mediated pathway. The production of GX results in a decreased efficiency of cellobiose fermentation (Figure 1), especially in the absence of a xylose-consumption pathway. The decrease in extracellular xylose concentration (Figure 1C) indicates that xylose is transported into the cell, likely via endogenous hexose transporters. Though lacking a xylose utilization pathway, the engineered yeast strain D452-2 expressing the pCS plasmid is expected to convert some xylose to xylitol by means of endogenous aldose reductase (Gre3) activity [21], consistent with the fact that xylitol was detected in the fermentation medium (Figure 1D). We further verified that much of the remaining xylose loss in the intracellular pool was due to its conversion to GX by the reverse phosphorolysis reaction catalyzed by CBP between G1P and xylose (Additional file 1: Figure S2). Interestingly, we found that the extracellular concentration of GX eventually starts to decrease, and at the same time that of xylose begins to recover (Figure $1 \mathrm{C}, \mathrm{E}$ ).

We propose that, in the presence of xylose, cellobiose is first transported into yeast cells via cellodextrin transporter mutant CDT-1 (F213L) and cleaved by CBP to generate glucose and G1P (Figure 6). At the same time, xylose is transported into the cell via hexose transporters. With xylose and G1P present inside the cell, CBP catalyzes the reverse phosphorolysis reaction, producing GX, which can be exported by the cellodextrin transporter (Figure 6). The model explains the initial decrease in xylose concentration and increase in GX concentration in the media (Figure $1 \mathrm{C}, \mathrm{E}$ ). CDT-1, a proton symporter [22], can reversibly transport substrates [4] because of the thermodynamic driving forces of high intracellular substrate (that is, GX) concentrations competing with high extracellular proton concentrations. At later stages of the fermentation, GX in the media is transported back into the cell via the cellodextrin transporter, along with the rest of the cellobiose (Figure 6). GX is then cleaved by SdCBP to generate G1P and xylose. Some xylose released is then exported back into the media via endogenous hexose transporters. The second part of the model explains the decrease in GX concentration and the increase in xylose concentration in the media (Figure 1C,E). 


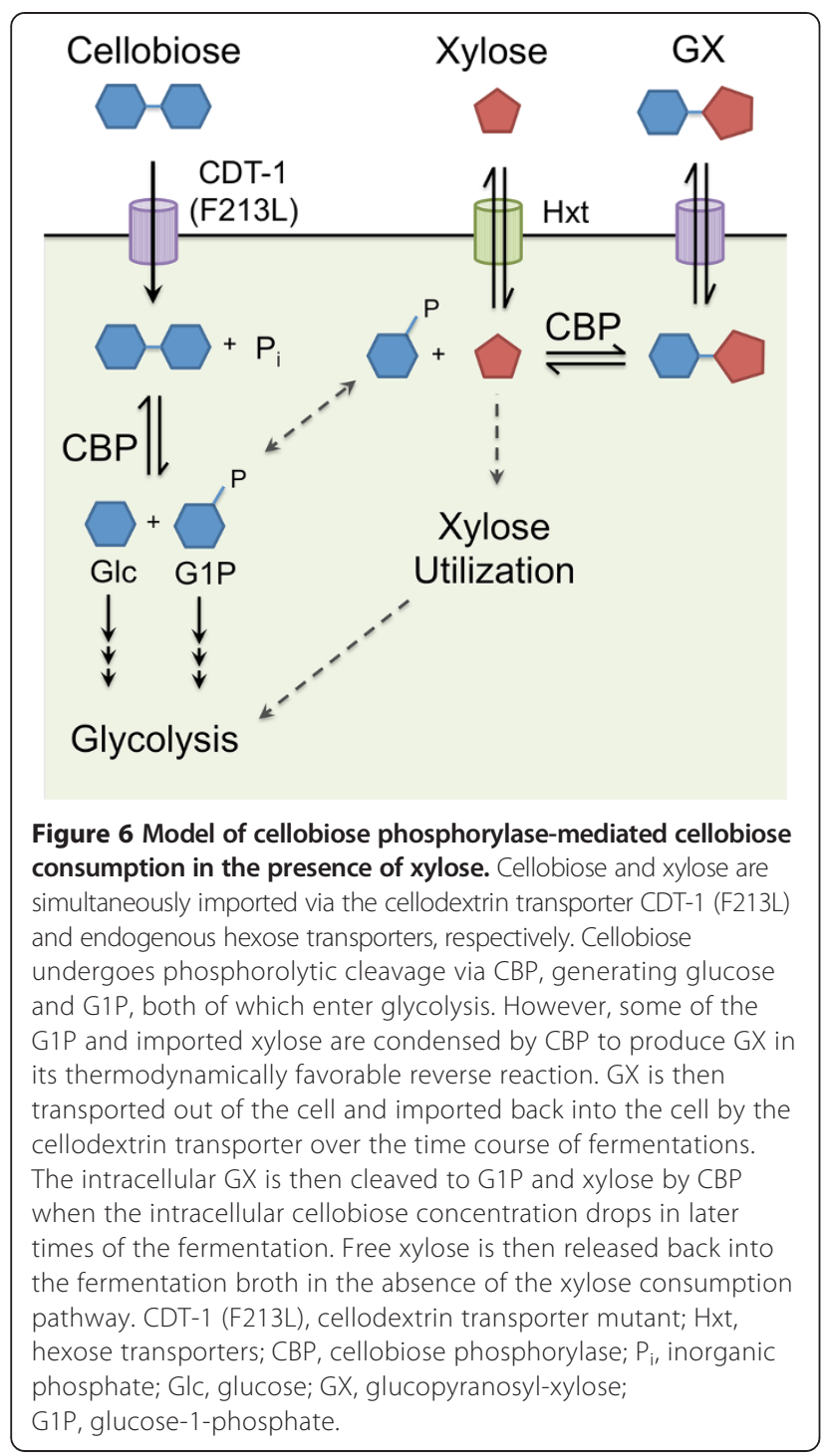

Formation and cleavage of GX as observed here is unfavorable to cellobiose fermentation. GX exhausts resources that could have been dedicated to cellobiose consumption, namely the use of CDT-1 to export and import GX and SdCBP to form and cleave GX. This is especially deleterious because GX processes occur simultaneously with cellobiose consumption (Figures 1A,E and 6). Furthermore, GX formation requires G1P as one of the substrates (Figure 2A). G1P is thus diverted from glycolysis, where in the absence of xylose it would be converted to G6P by phosphoglucomutase. GX formation would thereby decrease the rate of ethanol production because some of G1P produced from cellobiose via CBP is wasted in the formation of GX. These considerations suggest that GX formation likely results in a decrease in cellobiose import by CDT-1, slows cleavage of cellobiose, and reduces the rate of ethanol production. Extracellular concentrations of cellobiose and ethanol also support this model (Figure 1A,B).
The reported catalytic efficiency $\left(\mathrm{k}_{\text {cat,app }} / \mathrm{K}_{\mathrm{M} \text {,app }}\right)$ of $\mathrm{RaCBP}$ for xylose in the reverse phosphorolysis reaction is only $1 \%$ of that for glucose [18]. However, with SdCBP, a substantial amount of GX formation is observed (Figure 1E). This may be explained by differences between the two CBPs, or by the high concentrations of intracellular xylose and low concentrations of intracellular glucose present in our experiments. When the xylose utilization pathway is absent, high intracellular xylose concentrations are expected. Although we did not measure the intracellular concentration of xylose directly, it is expected to be similar to the extracellular concentration due to the fact that xylose is imported by hexose transporters, which are facilitators $[23,24]$. Thus, the imported xylose not accounted for by xylitol production (Figure 1C,D), would result in an intracellular concentration of xylose near or above the reported $\mathrm{K}_{\mathrm{M} \text {,app }}$ of RaCBP for xylose (25 mM) [18]. By contrast, the intracellular concentration of glucose is expected to be small because glucose can be efficiently converted to G6P and consumed by glycolysis. The maximal reported free intracellular glucose concentration is 2 to $3 \mathrm{mM}$ [25], slightly above the $\mathrm{K}_{\mathrm{M} \text {,app }}$ of RaCBP on glucose $(1.5 \mathrm{mM})$ [18]. Furthermore, the CBP reverse reaction is thermodynamically favorable, with a $\Delta G^{\circ}=-3.6 \mathrm{~kJ} \mathrm{~mol}^{-1}$ for cellobiose formation $[10,12]$. Thus, the amount of GX formation we observed was consistent with the known thermodynamic and kinetic properties of the enzymes used in the CBP-mediated cellobiose consumption pathway, especially due to the drive from a high intracellular xylose concentration.

By using in vitro competition assays, we identified $\mathrm{xy}$ lose as a mixed inhibitor of CBP for the cellobiose phosphorolytic reaction (Figure 3A,B). The synthesis of GX from xylose and G1P, albeit slow [26], shows that xylose can bind to the CBP enzyme active site (Figure 2). This helps to explain the decrease in the apparent affinity for cellobiose in the presence of xylose (increase in $K_{\mathrm{M} \text {,app}}$ ). The decrease in maximal phosphorolytic rate of cellobiose $\left(\mathrm{V}_{\max , \text { app }}\right)$ in the presence of xylose suggests that $x y-$ lose also inhibits cellobiose phosphorolytic activity in some other way, unrelated to xylose competition with cellobiose for the enzyme active site. CBP is a homodimer $[27,28]$, and its active site pocket is formed at the interface of an $(\alpha / \alpha)_{6}$-barrel domain and a helical extension from the N-terminal domain of the adjacent subunit (Figure 7) [28]. Notably, in a crystal structure of Cellulomonas uda CBP in complex with cellobiose [PDB: 3S4A] [29], the reducing end of the cellobiose molecule is in contact with the extension from the adjacent subunit (Figure 7). Xylose likely binds at this position, because its structure is similar to that of glucose, enabling the formation of GX from xylose and G1P. Thus, as xylose binds to and/or releases from the reducing end of the active site in one subunit, it may come into contact with the $\mathrm{N}$-terminal domain of the 


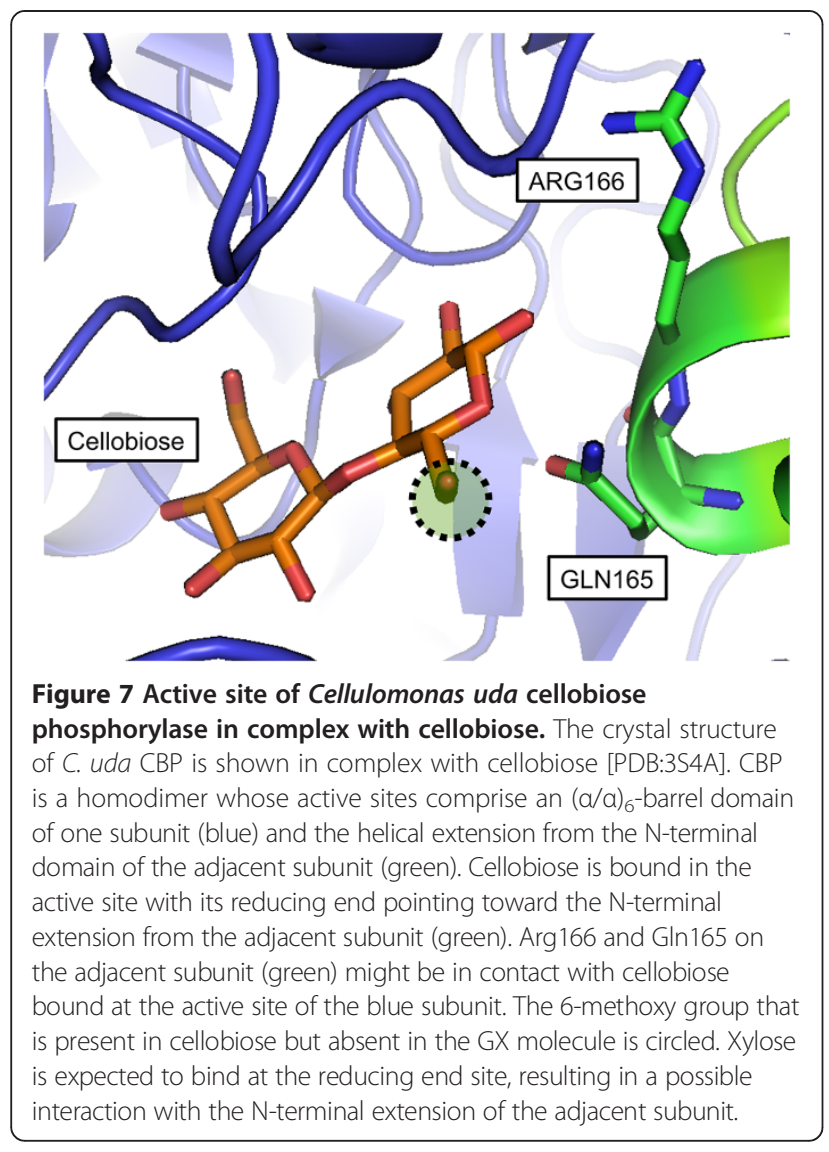

other subunit. The interaction may alter CBP enzymatic activity, preventing cellobiose phosphorylation in the adjacent unit or resulting in decreased product dissociation from the adjacent active site.

By carrying out cellobiose and xylose co-fermentation using an efficient xylose-utilizing strain, SR8-a [20], we were able to increase the cellobiose consumption rate and decrease GX titer (Figure 4A,B), likely by keeping the steady-state intracellular xylose concentration low. The low concentrations of xylose present inside the cell would improve the apparent kinetic properties of cellobiose phosphorylation, resulting in a faster cellobiose consumption rate (Figure 3). Furthermore, with less intracellular xylose present, less substrate is available for GX formation. Thus, smaller amounts of GX are made, exported out of the cell and re-imported, reserving the capacity of the cellodextrin transporter and CBP for cellobiose phosphorolysis (Figure 6). Finally, less GX formation allows G1P to more efficiently enter into glycolysis by its conversion to G6P, thereby increasing the ethanol production rate. Thus, an efficient xylose utilization pathway can be used to alleviate cellobiose fermentation inefficiencies due to the use of CBP.

For bacteria with CBP, ORFs encoding xylose isomerase are found to co-exist, suggesting that cellobiose and xylose co-fermentation by anaerobic bacteria may be common, for example in S. degradans, Cellvibrio gilvus, Ruminococcus sp. and Clostridium phytofermentans. Although co-fermentation has not been shown with these organisms, they may have evolved means of avoiding the production of GX. Previous efforts to construct an anaerobic cellobiose and xylose co-fermentation system in S. cerevisiae using CBP and xylose isomerase from Ruminococcus flavefaciens were only partly successful [16]. This may be due to the inefficient xylose isomerase conversion step, resulting in high intracellular concentrations of xylose that negatively impact CBP-mediated cellobiose consumption.

We also explored whether CBP-mediated cellobiose conversion in the presence of xylose could be augmented by the use of a hydrolytic enzyme to cleave GX after its formation. We found that the intracellular $\beta$-glucosidase GH1-1 from $N$. crassa was capable of GX hydrolysis to glucose and xylose (Figure 5). Thus, low levels of GH1-1 co-expressed with CBP might be used to reduce GX and its associated burdens on the cellobiose consumption pathway (Figure 6). However, cellobiose was preferred as a substrate for GH1-1 in comparison to GX (Table 3) and the catalytic efficiency of GH1-1 for cellobiose is higher than that of CBP for cellobiose [10]. Hence, co-expression of GH1-1 with CBP would likely result in most of the cellobiose being hydrolyzed to glucose instead of following the phosphorolytic pathway. This effect would defeat the purpose of using CBP for its energetic advantage, because G1P generation would be replaced by glucose production. To circumvent this challenge, the intracellular $\beta$ glucosidase would need to have an increased substrate specificity for GX and lower activity for cellobiose. Protein engineering of an intracellular $\beta$-glucosidase with these properties may be feasible, because xylose is smaller than glucose. Thus, the enzyme active site of $\beta$-glucosidase could be engineered to be more bulky, allowing the binding of GX while eliminating that of cellobiose. Successes in similar protein engineering challenges have been reported [30-32].

Alternatively, CBP could be engineered to reduce or eliminate GX production. This approach is advantageous because it addresses the GX complications directly. In contrast to the use of engineered GH1-1 for GX cleavage, this approach allows the system to fully harvest the energetic advantages of the phosphorolytic pathway. However, CBP protein engineering may be challenging because xylose is a mixed-inhibitor of CBP activity (Figure 3), and therefore may require random mutagenesis, multiple site saturation mutagenesis or evolutionary engineering approaches to achieve the necessary cellobiose specificity [30,33,34].

\section{Conclusions}

We have shown that xylose can have negative impacts on anaerobic cellobiose fermentation mediated by CBP 
in S. cerevisiae. Xylose can serve as a substrate along with G1P in a favorable reverse reaction to form the byproduct GX dimer. We have provided evidence that GX is likely exported out of cells and imported back by the exogenous cellodextrin transporter before being cleaved by SdCBP, exhausting resources that could have been reserved for cellobiose fermentation. Additionally, we identified xylose as a mixed-inhibitor of CBP activity, possibly due to the arrangement of enzyme active sites in the CBP homodimer. Cellobiose and xylose cofermentation by the efficient xylose-utilizing SR8-a strain increased the cellobiose fermentation rate and decreased GX formation, likely by maintaining a low intracellular xylose concentration. The intracellular $\beta$-glucosidase GH1-1 from $N$. crassa was also capable of cleaving GX, and could be used to augment the CBP-mediated cellobiose consumption pathway. However, the use of an intracellular $\beta$-glucosidase alongside $\mathrm{CBP}$ may require further protein engineering to improve the $\beta$-glucosidase specificity for GX over cellobiose.

\section{Methods}

\section{Plasmid construction}

Plasmids constructed and used in this study are listed in Table 1. Plasmids containing a codon-optimized CBP gene from S. degradans (SdCBP) [GenBank: 90020965] [10] and cellodextrin transporter mutant from $N$. crassa cdt-1 (F213L) [10] were used as templates for combining $c d t-1$ (F213L) and SdCBP expression cassettes in pRS426 (pCS). SdCBP was cloned into E. coli expression plasmid pET302 with an $\mathrm{N}$-terminal $\mathrm{His}_{6}$ tag to create pET-Sd. The InFusion HD Cloning Kit (Clontech, Mountain View, CA, USA) was used for all plasmid construction. Primers used are listed in Additional file 1: Table S1.

\section{Yeast strains and media}

S. cerevisiae background strains used in this study were D452-2 (MATa leu2 his3 ura3 can1) and SR8-a (ura3) (Table 1). Plasmids were transformed into these strains using a standard lithium acetate yeast transformation protocol [35]. Transformants were selected on synthetic defined medium plates, which contained DOBA (MP Biomedicals, Santa Ana, CA, USA) mixed with two-fold appropriate CSM dropout mixture.

\section{Fermentations}

Single colonies from synthetic defined plates were selected and re-streaked. Re-streaked colonies were inoculated in optimal minimal medium (oMM) supplemented with $20 \mathrm{~g} / \mathrm{L}$ of cellobiose to prepare seed cultures. The oMM contained $1.7 \mathrm{~g} / \mathrm{L}$ YNB Y1251 (Sigma, Saint Louis, MO, USA), two-fold appropriate CSM dropout mixture, $10 \mathrm{~g} / \mathrm{L}$ $\left(\mathrm{NH}_{4}\right)_{2} \mathrm{SO}_{4}, 1 \mathrm{~g} / \mathrm{L} \mathrm{MgSO}{ }_{4} 7 \mathrm{H}_{2} \mathrm{O}, 6 \mathrm{~g} / \mathrm{L} \mathrm{KH}_{2} \mathrm{PO}_{4}, 100 \mathrm{mg} / \mathrm{L}$ adenine hemisulfate, $10 \mathrm{mg} / \mathrm{L}$ inositol, $100 \mathrm{mg} / \mathrm{L}$ glutamic acid, $20 \mathrm{mg} / \mathrm{L}$ lysine, $375 \mathrm{mg} / \mathrm{L}$ serine and $0.1 \mathrm{M} 2-(\mathrm{N}-$ morpholino) ethanesulfonic acid (MES) pH 6.0. Seed cultures were harvested at mid- to late-exponential phase and washed twice with sterile water. Washed seed cultures were inoculated at an initial optical density at $600 \mathrm{~nm}$ of 20 in $200 \mathrm{~mL}$ serum flasks containing $50 \mathrm{~mL}$ of media. The flasks were closed with butyl rubber stoppers, sealed with aluminum crimps, and purged with nitrogen gas to obtain strict anaerobic fermentations. The fermentation media contained oMM supplemented with $80 \mathrm{~g} / \mathrm{L}$ cellobiose, with or without $40 \mathrm{~g} / \mathrm{L}$ xylose. The flasks were incubated at $30^{\circ} \mathrm{C}, 220 \mathrm{rpm}$. Extracellular concentrations of cellobiose, xylose, xylitol and ethanol were determined by high performance liquid chromatography on a Prominence HPLC (Shimadzu, Kyoto, Japan) equipped with Rezex RFQ-FastAcid H $10 \times 7.8 \mathrm{~mm}$ column. The column was eluted with $0.01 \mathrm{~N}$ of $\mathrm{H}_{2} \mathrm{SO}_{4}$ at a flow rate of $1 \mathrm{~mL} /$ min, $55^{\circ} \mathrm{C}$. Quantification of GX was performed using an ICS-3000 Ion Chromatography System (Dionex, Sunnyvale, CA, USA) equipped with a CarboPac ${ }^{\circ}$ PA200 carbohydrate column. The column was eluted with a $\mathrm{NaOAc}$ gradient in $100 \mathrm{mM} \mathrm{NaOH}$ at a flow rate of $0.4 \mathrm{~mL} / \mathrm{min}$, $30^{\circ} \mathrm{C}$.

\section{SdCBP protein purification}

pET-Sd (pET302-NT/His ${ }_{6}$-SdCBP) was transformed into the BL21 (DE3) E. coli strain and induced by isopropyl $\beta$ D-1-thiogalactopyranoside at a final concentration of $0.2 \mathrm{mM}$. E. coli cells were lysed and protein purified by His Bind Resin (Novagen, Darmstadt, Germany) according to supplied protocols. Purified SdCBP was stored in $20 \mathrm{mM}$ MES, pH 6.0 and quantified using a NanoDrop 1000 spectrophotometer, assuming an extinction coefficient of $1.79 \times 10^{5} \mathrm{M}^{-1} \mathrm{~cm}^{-1}, 280 \mathrm{~nm}$.

In vitro synthesis and purification of glucopyranosyl-xylose We incubated $10 \mathrm{mM}$ xylose and $10 \mathrm{mM}$ G1P with and without $20 \mathrm{nM}$ purified SdCBP in $20 \mathrm{mM}$ MES, pH 6.0 at $37^{\circ} \mathrm{C}$ for 12 hours. The reaction was stopped by dilution with $0.1 \mathrm{M} \mathrm{NaOH}$ at a ratio of 1:200. Signals of components in the solutions were detected using an ICS-3000 Ion Chromatography System (Dionex) with the same conditions described above.

The synthesized GX was purified by ÄKTA Purifier (GE Healthcare Life Sciences, Munich, Germany) equipped with a Supelclean ${ }^{\text {Tx }}$ ENVI-Carb ${ }^{\text {Tw }}$ column. The column was eluted with a gradient of acetonitrile at a flow rate of $3.0 \mathrm{~mL} / \mathrm{min}$ at room temperature. Purified fractions were verified using an ICS-3000 Ion Chromatography System (Dionex) with the same conditions described above.

\section{Mass spectrometry and tandem mass spectrometry}

MS of the GX in the in vitro synthesis solution was performed on an LTQ XL ion trap instrument (Thermo 
Fisher Scientific, San Jose, CA, USA) with an electrospray ionization source operated in negative mode. The sample was introduced into the mass spectrometer by direct injection into a flow of $50 \%$ water $/ 0.1 \%$ formic acid and $50 \%$ acetonitrile $/ 0.1 \%$ formic acid set at a flow rate of $0.2 \mathrm{~mL} / \mathrm{min}$. The MS settings were capillary temperature $350^{\circ} \mathrm{C}$, ion spray voltage $4 \mathrm{kV}$, sheath gas flow 60 (arbitrary units), auxiliary gas flow 10 (arbitrary units), sweep gas flow 5 (arbitrary units). The scan rate for full scan and MS/MS product ion scan was $\mathrm{m} / \mathrm{z} 95$ to $\mathrm{m} / \mathrm{z} 500$. The compound at $\mathrm{m} / \mathrm{z} 357$ was isolated with a $\mathrm{m} / \mathrm{z} 2$ isolation width $( \pm 1 \mathrm{Da})$ and fragmented with a normalized collision-induced dissociation energy setting of $35 \%$. The activation time and the activation $\mathrm{Q}$ were $30 \mathrm{~ms}$ and 0.250 , respectively. The mass measurement accuracy was $<3$ ppm root mean square.

\section{Competition assay and kinetic parameters}

We incubated $10 \mathrm{nM}$ of purified SdCBP, $5 \mathrm{mM}$ of inorganic phosphate and varying cellobiose concentrations at $30^{\circ} \mathrm{C}$ in $20 \mathrm{mM}$ MES, pH 6.0 with $0,2.5$ or $5 \mathrm{mM}$ xylose. All experiments were carried out in duplicate. G1P concentrations were detected continuously using a G1P Colorimetry Assay Kit (Sigma-Aldrich), according to the provided protocol. Initial rates at each cellobiose concentration were calculated from the rate of G1P production. Apparent kinetic parameters were determined by non-linear regression.

\section{In vitro glucopyranosyl-xylose hydrolytic activity assay}

GX synthesized as described above was used at a concentration of $1 \mathrm{mM}$. The substrate was incubated with $0.5 \mu \mathrm{M}$ of purified $\beta$-xylosidase (NCU01900) or $\beta$ glucosidase (NCU00130) in $1 \times \mathrm{PBS}, \mathrm{pH} 7.4$ at $30^{\circ} \mathrm{C}$ for 2 hours. $\beta$-xylosidase (NCU01900) (unpublished observations by $\mathrm{Dr}$. Xin Li) and $\beta$-glucosidase (NCU00130) were expressed and purified as described [6]. To stop the reactions, they were diluted with $0.1 \mathrm{M} \mathrm{NaOH}$. Signals of components in the solutions were detected using an ICS-3000 Ion Chromatography System (Dionex) with the same conditions described above.

For kinetic parameter comparisons, $20 \mathrm{nM}$ of purified GH1-1 and varying concentrations of cellobiose and purified GX were incubated at $30^{\circ} \mathrm{C}$ in $1 \times \mathrm{PBS}, \mathrm{pH}$ 7.4. All reactions were carried out in duplicate. The reactions were stopped at $0,5,10$ and 15 minutes by addition of $0.1 \mathrm{M} \mathrm{NaOH}$. Initial rates at each cellobiose and GX concentration were calculated from the rate of glucose production. The ICS-3000 Ion Chromatography System (Dionex) equipped with CarboPac ${ }^{\mathrm{TM}}$ PA20 column was eluted with $3 \mathrm{mM} \mathrm{KOH}$ at a flow rate of $0.4 \mathrm{~mL} / \mathrm{min}$, $30^{\circ} \mathrm{C}$, for glucose and xylose separation and to determine glucose concentrations in the reactions. Apparent kinetic parameters were determined by non-linear regression.

\section{Additional file}

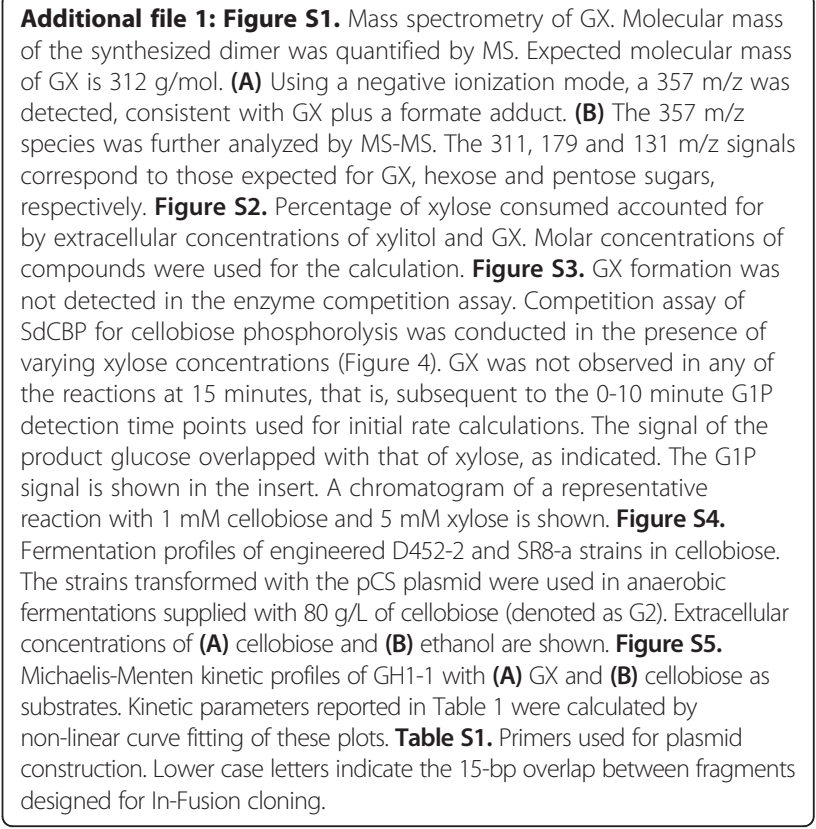

\section{Abbreviations}

CBP: cellobiose phosphorylase; CDT-1: cellodextrin transporter from Neurospora crassa; CDT-1 (F213L): mutant of cellodextrin transporter from N. crassa; G1P: glucose-1-phosphate; G6P: glucose-6-phosphate; GH1-1: beta-glucosidase; GX: glucopyranosyl-xylose; MES: 2-(N-morpholino) ethanesulfonic acid; MS: mass spectrometry; MS-MS: tandem mass spectrometry; oMM: optimal minimal media; ORF: open reading frame; PBS: phosphate buffered saline; RaCBP: cellobiose phosphorylase from Ruminococcus albus NE1; SdCBP: cellobiose phosphorylase from Saccharophagus degradans.

\section{Competing interests}

The authors declare that they have no competing interests.

\section{Authors' contributions}

KC and JHDC designed all experiments. KC, VK and AEG performed fermentation experiments. KC and XL prepared and purified enzymes and substrates. KC performed enzymatic assay experiments. SB performed mass spectrometry experiments. SJH, EJO, JMG and YSJ shared their preliminary observations of possible GX formation in fermentations. KC and JHDC analyzed and wrote the manuscript. All authors read and approved the manuscript.

\section{Acknowledgements}

This work was supported by funding from the Energy Biosciences Institute to YSJ and JHDC. The authors thank Dr Soo Rin Kim for generously providing S. cerevisiae SR8-a, and Dr Yuping Lin, Dr Ligia Acosta-Sampson, and Dr Owen W Ryan for helpful discussions.

\section{Author details}

${ }^{1}$ Department of Plant and Microbial Biology, University of California, Berkeley, CA, USA. ${ }^{2}$ Department of Chemistry, University of California, Berkeley, CA, USA. ${ }^{3}$ Department of Molecular and Cell Biology, University of California, Berkeley, CA, USA. ${ }^{4}$ Energy Biosciences Institute, University of California, Berkeley, CA, USA. ${ }^{5}$ Department of Food Science and Human Nutrition, University of Illinois, Urbana, IL, USA. Institute for Genomic Biology, University of Illinois, Urbana, IL, USA. ${ }^{7}$ Department of Bioengineering and Technology, Kangwon National University, Chuncheon, Republic of Korea. ${ }^{8}$ Physical Biosciences Division, Lawrence Berkeley National Laboratory, Berkeley, CA, USA.

Received: 19 February 2014 Accepted: 21 May 2014

Published: 7 June 2014 


\section{References}

1. Carroll A, Somerville C: Cellulosic biofuels. Annu Rev Plant Biol 2009, 60:165-182.

2. Hinman ND, Wright JD, Hoagland W, Wyman CE: Xylose fermentation-an economic analysis. Appl Biochem Biotechnol 1989, 20/21:391-401.

3. Galbe M, Zacchi G: A review of the production of ethanol from softwood. Appl Microbiol Biotechnol 2002, 59:618-628.

4. Ha SJ, Galazka JM, Kim SR, Choi JH, Yang X, Seo JH, Glass NL, Cate JH, Jin YS: Engineered Saccharomyces cerevisiae capable of simultaneous cellobiose and xylose fermentation. Proc Natl Acad Sci U S A 2011, 108:504-509.

5. Li S, Du J, Sun J, Galazka JM, Glass NL, Cate JH, Yang X, Zhao H: Overcoming glucose repression in mixed sugar fermentation by co-expressing a cellobiose transporter and a beta-glucosidase in Saccharomyces cerevisiae. Mol Biosyst 2010, 6:2129-2132.

6. Galazka JM, Tian C, Beeson WT, Martinez B, Glass NL, Cate JH: Cellodextrin transport in yeast for improved biofuel production. Science 2010, 330:84-86.

7. Tantirungkij M, Nakashima N, Seki T, Yoshida T: Construction of xyloseassimilating Saccharomyces cerevisiae. J Ferm Bioeng 1993, 75:83-88.

8. Kötter P, Ciriacy M: Xylose fermentation by Saccharomyces cerevisiae. Appl Microbiol Biotechnol 1993, 38:776-783.

9. Kuyper M, Winkler AA, van Dijken JP, Pronk JT: Minimal metabolic engineering of Saccharomyces cerevisiae for efficient anaerobic xylose fermentation: a proof of principle. FEMS Yeast Res 2004, 4:655-664.

10. Ha SJ, Galazka JM, Joong Oh E, Kordic V, Kim H, Jin YS, Cate JH: Energetic benefits and rapid cellobiose fermentation by Saccharomyces cerevisiae expressing cellobiose phosphorylase and mutant cellodextrin transporters. Metab Eng 2013, 15:134-143.

11. Lou J, Dawson KA, Strobel HJ: Role of phosphorolytic cleavage in cellobiose and cellodextrin metabolism by the ruminal bacterium Prevotella ruminicola. Appl Environ Microbiol 1996, 62:1770-1773.

12. Alexander JK: Characteristics of cellobiose phosphorylase. J Bacteriol 1961, 81:903-910.

13. Goldberg RN: Thermodynamics of hexokinase-catalyzed reactions. Biophys Chem 1975, 3:192-205.

14. Bro C, Knudsen S, Regenberg B, Olsson L, Nielsen J: Improvement of galactose uptake in Saccharomyces cerevisiae through overexpression of phosphoglucomutase: example of transcript analysis as a tool in inverse metabolic engineering. Appl Environ Microbiol 2005, 71:6465-6472.

15. de Kok S, Kozak BU, Pronk JT, van Maris AJ: Energy coupling in Saccharomyces cerevisiae: selected opportunities for metabolic engineering. FEMS Yeast Res 2012, 12:387-397.

16. Aeling KA, Salmon KA, Laplaza JM, Li L, Headman JR, Hutagalung AH, Picataggio S: Co-fermentation of xylose and cellobiose by an engineered Saccharomyces cerevisiae. J Ind Microbiol Biotechnol 2012, 39:1597-1604.

17. Rutter C, Chen R: Improved cellobiose utilization in $E$. coli by including both hydrolysis and phosphorolysis mechanisms. Biotechnol Lett 2014, 36:301-307.

18. Hamura K, Saburi W, Abe S, Morimoto N, Taguchi H, Mori H, Matsui H: Enzymatic characteristics of cellobiose phosphorylase from Ruminococcus albus NE1 and kinetic mechanism of unusual substrate inhibition in reverse phosphorolysis. Biosci Biotechnol Biochem 2012, 76:812-818

19. Hosaka K, Nikawa J, Kodaki T, Yamashita S: A dominant mutation that alters the regulation of INO1 expression in Saccharomyces cerevisiae. J Biochem 1992, 111:352-358.

20. Kim SR, Skerker JM, Kang W, Lesmana A, Wei N, Arkin AP, Jin YS: Rational and evolutionary engineering approaches uncover a small set of genetic changes efficient for rapid xylose fermentation in Saccharomyces cerevisiae. PLoS One 2013, 8:e57048

21. Traff KL, Otero Cordero RR, van Zyl WH, Hahn-Hagerdal B: Deletion of the GRE3 aldose reductase gene and its influence on xylose metabolism in recombinant strains of Saccharomyces cerevisiae expressing the xylA and XKS1 genes. Appl Environ Microbiol 2001, 67:5668-5674.

22. Kim H, Lee WH, Galazka JM, Cate JH, Jin YS: Analysis of cellodextrin transporters from Neurospora crassa in Saccharomyces cerevisiae for cellobiose fermentation. Appl Microbiol Biotechnol 2014, 98:1087-1094.

23. Hamacher T, Becker J, Gardonyi M, Hahn-Hagerdal B, Boles E: Characterization of the xylose-transporting properties of yeast hexose transporters and their influence on xylose utilization. Microbiology 2002, $148: 2783-2788$.
24. Ozcan S, Johnston M: Function and regulation of yeast hexose transporters. Microbiol Mol Biol Rev MMBR 1999, 63:554-569.

25. Teusink B, Diderich JA, Westerhoff HV, van Dam K, Walsh MC: Intracellular glucose concentration in derepressed yeast cells consuming glucose is high enough to reduce the glucose transport rate by $50 \%$.J Bacteriol 1998, 180:556-562.

26. Kitaoka M, Taniguchi H, Sasaki T: Production of glucosyl-xylose using cellvibrio-gilvus cells and its properties. Appl Microbiol Biotechnol 1990, 34:178-182.

27. Kitaoka M, Sasaki T, Taniguchi H: Purification and properties of laminaribiose phosphorylase (EC 2.4 1.31) from Euglena gracilis Z. Arch Biochem Biophys 1993, 304:508-514.

28. Bianchetti CM, Elsen NL, Fox BG, Phillips GN Jr: Structure of cellobiose phosphorylase from Clostridium thermocellum in complex with phosphate. Acta Crystallogr Sect F Struct Biol Cryst Commun 2011, 67:1345-1349.

29. Van Hoorebeke A, Stout J, Kyndt J, De Groeve M, Dix I, Desmet T, Soetaert W, van Beeumen J, Savvides SN: Crystallization and X-ray diffraction studies of cellobiose phosphorylase from Cellulomonas uda. Acta Crystallogr Sect $F$ Struct Biol Cryst Commun 2010, 66:346-351.

30. Antikainen NM, Martin SF: Altering protein specificity: techniques and applications. Bioorg Med Chem 2005, 13:2701-2716.

31. DeSantis G, Liu J, Clark DP, Heine A, Wilson IA, Wong CH: Structure-based mutagenesis approaches toward expanding the substrate specificity of D-2-deoxyribose-5-phosphate aldolase. Bioorg Med Chem 2003, 11:43-52.

32. Cronin CN: Redesign of choline acetyltransferase specificity by protein engineering. J Biol Chem 1998, 273:24465-24469.

33. Wang JX, Zhang SF, Tan HD, Zhao ZB: PCR-based strategy for construction of multi-site-saturation mutagenic expression library. J Microbiol Meth 2007, 71:225-230.

34. Chica RA, Doucet N, Pelletier JN: Semi-rational approaches to engineering enzyme activity: combining the benefits of directed evolution and rational design. Curr Opin Biotech 2005, 16:378-384.

35. Gietz RD, Woods RA: Transformation of yeast by lithium acetate/singlestranded carrier DNA/polyethylene glycol method. Methods Enzymol 2002, 350:87-96.

doi:10.1186/1754-6834-7-85

Cite this article as: Chomvong et al:: Overcoming inefficient cellobiose fermentation by cellobiose phosphorylase in the presence of xylose. Biotechnology for Biofuels 2014 7:85

\section{Submit your next manuscript to BioMed Central and take full advantage of:}

- Convenient online submission

- Thorough peer review

- No space constraints or color figure charges

- Immediate publication on acceptance

- Inclusion in PubMed, CAS, Scopus and Google Scholar

- Research which is freely available for redistribution 